\title{
Motor Commands Induce Time Compression for Tactile Stimuli
}

\author{
Alice Tomassini, ${ }^{1}$ Monica Gori, ${ }^{1}$ Gabriel Baud-Bovy, ${ }^{1,2}{ }^{-}$Giulio Sandini, ${ }^{1}$ and Maria Concetta Morrone ${ }^{3,4}$ \\ ${ }^{1}$ Department of Robotics, Brain and Cognitive Sciences, Istituto Italiano di Tecnologia, 16163 Genova, Italy, ${ }^{2}$ Faculty of Psychology, Università Vita-Salute \\ San Raffaele, 20132 Milano, Italy, ${ }^{3}$ Department of Translational Research on New Technologies in Medicine and Surgery, Università di Pisa, 56123 Pisa, \\ Italy, and ${ }^{4}$ Scientific Institute Stella Maris, 56018 Calambrone, Pisa, Italy
}

\begin{abstract}
Saccades cause compression of visual space around the saccadic target, and also a compression of time, both phenomena thought to be related to the problem of maintaining saccadic stability (Morrone et al., 2005; Burr and Morrone, 2011). Interestingly, similar phenomena occur at the time of hand movements, when tactile stimuli are systematically mislocalized in the direction of the movement (Dassonville, 1995; Watanabe et al., 2009). In this study, we measured whether hand movements also cause an alteration of the perceived timing of tactile signals. Human participants compared the temporal separation between two pairs of tactile taps while moving their right hand in response to an auditory cue. The first pair of tactile taps was presented at variable times with respect to movement with a fixed onset asynchrony of $150 \mathrm{~ms}$. Two seconds after test presentation, when the hand was stationary, the second pair of taps was delivered with a variable temporal separation. Tactile stimuli could be delivered to either the right moving or left stationary hand. When the tactile stimuli were presented to the motor effector just before and during movement, their perceived temporal separation was reduced. The time compression was effector-specific, as perceived time was veridical for the left stationary hand. The results indicate that time intervals are compressed around the time of hand movements. As for vision, the mislocalizations of time and space for touch stimuli may be consequences of a mechanism attempting to achieve perceptual stability during tactile exploration of objects, suggesting common strategies within different sensorimotor systems.
\end{abstract}

Key words: action; sensory-motor; time perception; touch

\section{Introduction}

The external world is mainly sensed through active exploration (Gibson, 1962), and awareness of one's own movement is fundamental to attribute sensory stimulation to its true physical source (Helmholtz, 1866).

In recent years the inherent interdependence between sensory and motor functions has been recognized as a general and indisputable operating principle in the brain, extending the notion of "purely sensory" to encompass also the implicit role of motor signals: meaningful information about the spatial and temporal arrangement of events in the external world cannot be gained unless sensory information is integrated with a representation of one's own body and its movement (Vaziri et al., 2006; Rucci et al., 2007; Medendorp, 2011; Yoshioka et al., 2011). The haptic sense is an exemplar case of sensory-motor integrative processes. Tactile events uniquely occur on the body surface and most often as a result of an active movement of the body. To accurately locate

\footnotetext{
Received July 1, 2013; revised May 26, 2014; accepted May 31, 2014.

Author contributions: A.T., M.G., G.B.-B., G.S., and M.C.M. designed research; A.T. performed research; A.T. and G.B.-B. analyzed data; A.T. and M.C.M. wrote the paper.

This work was supported by PF7-EC projects XPERIENCE, ERC-STANIB, and ERC-ECSPLAIN. We thank Marco Jacono for his help in building the setup and preparing the experiments.

The authors declare no competing financial interests.

Correspondence should be addressed to Dr Alice Tomassini, Department of Robotics, Brain and Cognitive Sciences (RBCS), Istituto Italiano di Tecnologia (IIT), Via Morego 30, 16163 Genova, Italy. E-mail: alice.tomassini@iit.it.

DOI:10.1523/JNEUROSCI.2782-13.2014

Copyright $\odot 2014$ the authors $\quad 0270-6474 / 14 / 349164-09 \$ 15.00 / 0$
}

tactile events in space and time somatotopic codes must be dynamically updated through integration with proprioceptive and motor information. Internally generated action-signals might provide predictions about the location of the stimulated body site in space as well as about the timing of stimulation allowing to coping with delays and variability in sensory transmission. However, a temporal mismatch between the prediction and the actual movement could cause perceptual alterations that have been observed in both vision and touch.

Visual stimuli flashed just before and during the execution of saccades are mislocalized toward the saccadic target, inducing de facto a compression of space along the direction of the movement (Morrone et al., 1997; Ross et al., 1997; Lappe et al., 2000). Complex distortions affect not only space but also time, and do so with a similar time course tightly locked to eye movement execution (Morrone et al., 2005; Binda et al., 2009). Apparent event time is both shifted and compressed so that the temporal separation of two visual stimuli is reduced by half of its physical length and even their order of appearance sometimes reversed.

These findings are thought to be interconnected and be the expression of a predictive mechanism, grounded on interlaced representations of space and time, that probably absolves the complex role of visual perceptual stability (Burr and Morrone, 2011). Distortions of time are also linked to the internal body schema representation and can be so strong to induce a reversal in the apparent order of tactile events (Yamamoto and Ki- 
A

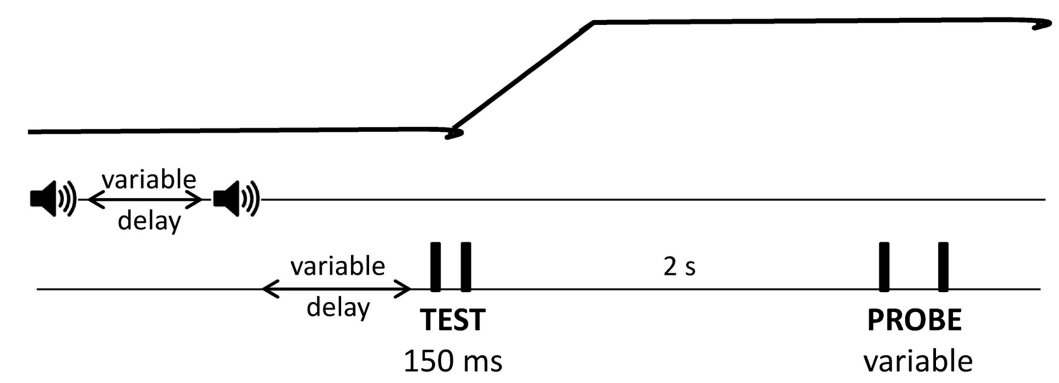

B
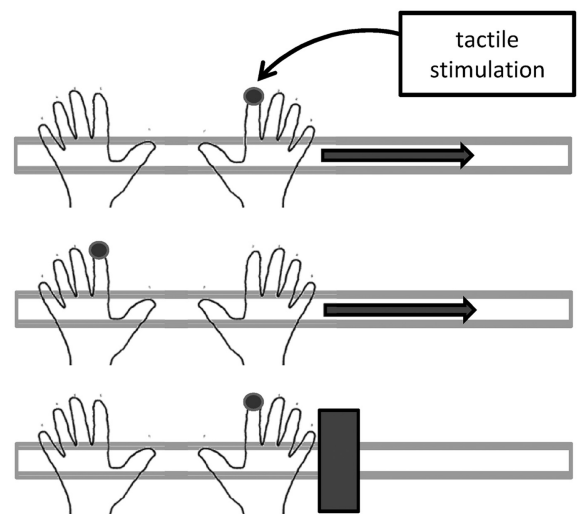

ISOMETRIC CONTRACTION

Figure 1. Experimental paradigm and conditions. $\boldsymbol{A}$, Schematic representation of the movement of the hand and of the relevant sensory events occurring during each trial: all events are aligned in time. Each trial starts with the presentation of an acoustic tone (start signal); after a variable pause a second tone is presented (go signal) that instructs participants to move their right hand as fast as possible. The first pair of tactile taps (test) is presented at variable delays from the go signal, with a fixed onset asynchrony of 150 $\mathrm{ms}$. After $2 \mathrm{~s}$ from test presentation the second pair of taps is presented (probe) with a variable temporal separation. $\boldsymbol{B}$, Experimental conditions. The movement condition implies the movement of the right hand; tactile stimulations are delivered either to the right moving hand or to the left static hand with equal probability across trials. The isometric contraction condition implies the generation of a transient lateral force against a block, with no movement execution; tactile stimulation is always delivered to the right contracting hand.

tazawa, 2001a,b), similarly to what has been shown for perisaccadic vision (Binda et al., 2009).

Interestingly, some data suggest that similar phenomena also occur around the time of hand movements, when tactile stimuli are systematically mislocalized in the direction of the movement (Dassonville, 1995; Watanabe et al., 2009; Maij et al., 2011) and perceptually delayed with respect to the subjective onset time of the movement (Dassonville, 1995; Jackson et al., 2011). This suggests that similar strategies may be exploited by different sensorimotor systems during the active exploration of the environment.

In this study, we adopted a paradigm similar to that previously used in vision (Morrone et al., 2005) and measured whether hand movements cause an alteration of the perceived timing of tactile signals.

\section{Materials and Methods}

Apparatus

Participants sat in front of a table with both hands resting on two handshaped plaster casts. The cast for the right hand was mounted on a sliding guide (length, $1.5 \mathrm{~m}$ ) so that its movement was constrained along one direction.

Tactile stimulations were delivered by means of two solenoid tappers (diameter $8 \mathrm{~mm}$, weight $2 \mathrm{~g}$; MST3 Miniature Solenoid Tappers, M\&E Solve) securely fixed on the right and left index finger pads with a Velcro strap.

The movement of the right hand was recorded by means of motion tracking cameras (Optotrak Certus Motion Capture System, Northern
Digital) that measured the position of one marker, attached to the nail of the right index finger, at a sampling rate of $200 \mathrm{~Hz}$.

To determine the time instant when the tactile stimulations were presented relative to the movement of the hand, a third tapper was operated in synchrony with the stimulating tappers and the movement of its pin was also recorded by the Optotrak system and used as a time stamp.

Forces and torques produced by the right hand were also measured by a six-axis force/ torque transducer (Mini40 F/T transducer; ATI Industrial Automation) fixed to the lower surface of the plaster cast.

\section{Procedure}

Experiment 1: temporal judgments during movement. Participants were asked to compare the temporal separation between two pairs of tactile taps while performing rapid movements with their right arm. An auditory tone (frequency $440 \mathrm{~Hz}$; duration $50 \mathrm{~ms}$ ) marked the beginning of each trial. After a variable interval (ranging from 0.8 to $1.8 \mathrm{~s}$ ) a second, identical auditory tone cued participants to move their right hand as fast as possible. At variable delays from the sound presentation (from $\sim 0$ to $\sim 700 \mathrm{~ms}$ after the sound) the first pair of taps (the test) was delivered to the right/left index finger with a fixed temporal separation of 150 ms. Two seconds after the test presentation the second pair of taps (the probe) was delivered to the same hand, with a variable separation ranging from 50 to $250 \mathrm{~ms}$ (Fig. $1 \mathrm{~A}$; schematic illustration of the experimental paradigm). The intensity of each tactile tap was $2.5 \mathrm{~V}$ (corresponding to a peak force of $27.5 \mathrm{~g}$ as measured with a precision scale) and its duration $8 \mathrm{~ms}$. To check that the actual stimulus timing corresponded to that commanded via computer we recorded the time course of the pin displacement by means of an analogic accelerometer (attached on top of the tapper surface) at a sampling rate of $5000 \mathrm{~Hz}$. The timing of the recorded acceleration profile matched the nominal stimulus timing $(8 \mathrm{~ms})$. Participants indicated verbally which pair of taps was separated by the longer time interval. Participants were required to keep their eyes closed and to wear noise-reducing headphones and earplugs that according to the self-reports of all subjects were sufficient to mask the noise produced by the tactile stimulators without preventing from hearing the acoustic signals.

Before performing the experiment participants underwent a training phase during which they learned to execute rightward hand movements of $\sim 450 \mathrm{~ms}$ in duration and $15 \mathrm{~cm}$ in amplitude in response to the auditory "go" signal. Approximately 20 hand movements were sufficient for all participants to achieve a stable performance during training. The practice trials were also used to evaluate the subject's mean reaction time to appropriately set the presentation times of the test stimulus so that sampling was mostly concentrated in a temporal window comprised between $200 \mathrm{~ms}$ before to $200 \mathrm{~ms}$ after movement onset time.

Hand movements were checked on a trial-by-trial basis by the experimenter and trials were discarded in case of incorrect movement execution; an automated procedure also rejected trials when the reaction time and movement duration were $<50$ and $150 \mathrm{~ms}$, respectively. To control the detectability of both taps in the test pair, we asked subjects to report whether they perceived one or two stimuli in each trial and discarded all trials where unclear percepts occurred. Overall, the number of rejected trials did not exceed 3\% for each subject and condition. 
We tested two different conditions (Fig. 1B): the movement condition where participants were required to move their right arm and the isometric contraction condition where participants produced a horizontal force with their right arm with actual displacement impeded by a block. In the movement condition, the two pairs of tactile taps were both presented randomly to the right or to the left index finger with equal probability of stimulating either hand while in the isometric contraction condition tactile stimuli were always delivered to the right index finger.

Data were collected on eight participants (mean age $=26.28 \pm 1.5$ years; two males; one author). Seven subjects were tested on the movement condition. Three subjects from the previous group plus one author performed also the isometric contraction condition. All subjects completed at least 16 sessions for the movement and seven sessions for the isometric contraction condition of 60 trials each.

Experiment 2: control for tactile suppression. To control for the effects of tactile suppression during movement on perceived time we performed an intensity-matching experiment. First, we measured the amount of tactile attenuation during movement. Each stimulus consisted in a single tactile tap, instead of a pair of taps; the test stimulus, delivered just before or during movement, had fixed intensity of $2.5 \mathrm{~V}$ (equal to the intensity of the stimuli used in Experiment 1), whereas the probe, delivered after the movement when the hand was stationary again, varied in intensity on a trial-by-trial basis (from 1.4 to $3 \mathrm{~V}$ ). Subjects had to report verbally which of the two stimuli was perceived as more intense. We then repeated the time perception experiment in stationary conditions (with neither hand movement nor contraction) manipulating the intensity of the stimuli delivered to the right resting hand according to the results obtained in the above described intensity-matching condition. Two conditions were randomly intermingled: in the baseline condition, the test stimulus (150 $\mathrm{ms}$ ) and the probe (variable temporal separation) had the same physical intensity of $2.5 \mathrm{~V}$; in the matched condition, the probe always had an intensity of $2.5 \mathrm{~V}$, whereas the intensity of the test was matched, separately for each subject, to the lowest intensity perceived during movement. We tested five of the seven naive subjects who had already taken part in Experiment 1.

Given the objective two-alternative forced choice design (2AFC), we planned five subjects for each experiment and condition. Unfortunately, only three participants of the original group (Experiment 1) gave their availability to complete also the isometric contraction condition. Thus, we decided to include one of the authors (A.T.) for this condition. To allow for the appropriate within-subject comparisons the author completed both the movement (with stimuli delivered only to the moving hand) and the isometric condition.

\section{Data analysis}

Data analysis was performed off-line. Movement onset time was determined by an automated algorithm as the instant corresponding to the fifth frame of a series of 10 consecutive frames where the first derivative of the velocity of the hand was greater than zero. The same computation was applied to the force produced along the axis parallel to the direction of the movement to yield force onset time. This force component was chosen as it was aligned with the direction along which the participants pushed. The end of the movement was determined as the instant of time when hand velocity became $<10 \mathrm{~mm} / \mathrm{s}$.

The time of stimulus presentation with respect to hand movement was determined by applying a velocity threshold $(15 \mathrm{~mm} / \mathrm{s})$ to the movement of the tapper triggered in synchrony with the first tap of the test pair and recorded by the Optotrak system. The time of stimulus presentation with respect to hand force was determined by acquiring jointly the voltage signal delivered to the tapper controller and the data from the force sensor by means of a national instrument data acquisition device (sampling rate $1000 \mathrm{~Hz}$ ). Test presentation times were then expressed relative to the central point of the temporal interval $(150 \mathrm{~ms})$ marked by the test pair. Stimulus latencies were calculated as the difference between test presentation time and movement (for the movement condition) or force (for the isometric contraction condition and for the intensity-matching control experiment) onset time. Thus, negative and positive latency values indicate that the center of the test interval fell before and after the onset of hand movement/contraction, respectively.

Data were grouped in different bins according to stimulus latency and then fitted separately with cumulative Gaussian functions, estimated by means of the Maximum Likelihood method. Bin size was chosen so that psychometric functions were never fitted to datasets with $<30$ trials. Approximately 50 trials served on average to calculate each psychometric function.

Both the Point of Subjective Equality (PSE) and the Just Noticeable Difference threshold [corresponding to the standard deviation (SD) of the fitted cumulative Gaussian function] were derived from the psychometric function parameters. The standard errors (SEs) of the PSEs and SDs were estimated by bootstrap.

The above described analysis requires a minimum number of trials $(\sim 30)$ to have reliable fitting of the psychometric functions, constraining the size and position of the latency bins.

To be able to evaluate on statistical basis the relation between perceived time (PSE) and stimulus latency taking into account the different number of trials collected for each subject and stimulus presentation time we analyzed the pool of single trials using the powerful generalized linear mixed model (GLMM) analysis (Moscatelli et al., 2012). The GLMM analysis was conducted after data collection for the entire subject pool was completed. We applied GLMM with a logit link function and a Bernoulli distribution. With this analysis, a single model is fitted for all subjects, taking into account the individual variability in the responses and the different number of observations (trials) collected for each subject. To keep complexity at a reasonable level, separate GLMM analyses were conducted for the moving and stationary hand including the whole 
A

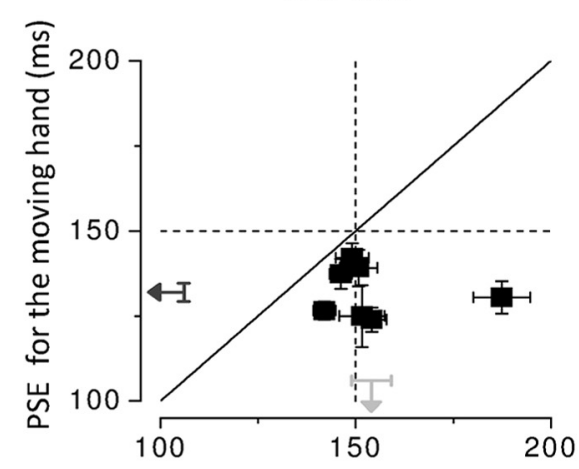

$+50 \mathrm{~ms}$

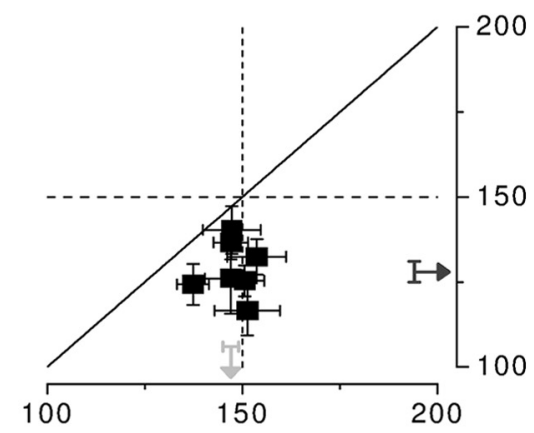

PSE for the static hand (ms)

B

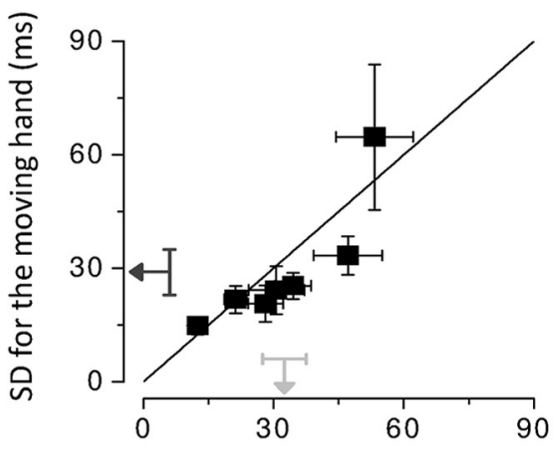

SD for the static hand (ms)

C Perceived time

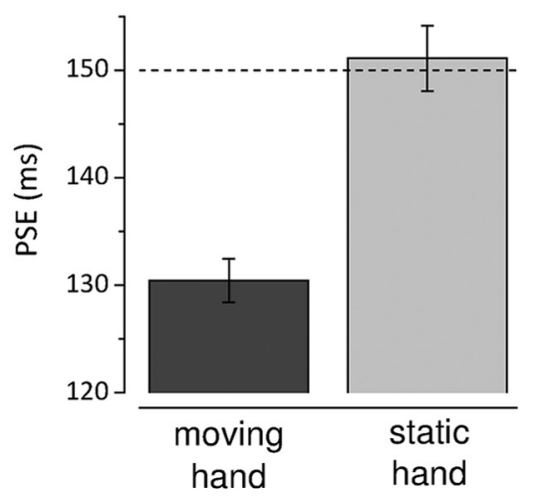

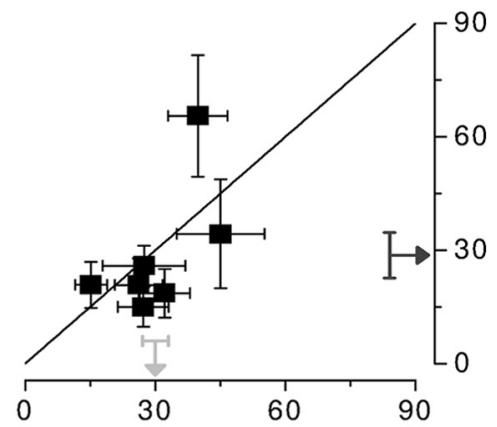

90
200

factors ( $u$ i0 and uil in Eq. 1) were selected by comparing various covariance structures and choosing the model with the lowest Akaike Information Criterion, which takes into account the goodness of fit and the number of parameters (the complexity) of the model.

\section{Results}

Experiment 1: time compression during movement

Data for two representative subjects are shown in Figure 2 for three critical stimulus presentation times relative to hand movement onset. The temporal interval estimation varies whether the stimulus is presented long before, close or during movement execution and whether on the moving or on the stationary hand. Well before movement initiation $(-170 \mathrm{~ms}$; Fig. 2, left), time perception is almost accurate for stimuli presented to either hand. The fitted psychometric functions from the moving and static hands are nearly overlapped, with PSEs approaching the actual time interval $(150 \mathrm{~ms})$ in both cases. When the tactile taps are presented to the right moving hand close to movement onset ( $-40 \mathrm{~ms}$; Fig. 2, middle), their apparent temporal separation is reduced by $\sim 30 \mathrm{~ms}$, yielding PSEs of $121 \pm 4 \mathrm{~ms}$ (SE) and $124 \pm 3 \mathrm{~ms}$ (SE) for subjects SC and LD, respectively. A similar compression of time is observed when the stimuli are presented just after the movement has started (+40 ms; Fig. 2, right).

We find that for all participants perceived duration in the epoch shortly preceding $(-50 \mathrm{~ms})$ and following movement onset $(+50 \mathrm{~ms})$ is very different between the two hands (Fig. 3A). All PSEs for the moving hand are $<150 \mathrm{~ms}$, whereas they are clustered $\sim 150 \mathrm{~ms}$ for the static hand. In other words, perceived time is compressed for the right moving hand while almost veridical for the left stationary hand. Importantly, we also find that the task is performed with the same precision (SD) for both hands, notwithstanding whether time is compressed or veridical (Fig. 3B), indicating that movement preparation and execution do not impair temporal judgments.

The PSEs averaged over the temporal window between -100 and $+100 \mathrm{~ms}$ are set of trials with stimulus latencies up to $400 \mathrm{~ms}$ before movement onset. The model included probe (stimulus temporal separation, probe $_{i j}$ for subject $i$ and trial $j$ ) and latency (lat $t_{i j}$ ) as fixed effects and subject and probe as random factors as follows:

$$
Y i j=\beta 0+u i 0+(\beta 1+u i 1) \text { probeij }+\beta 2 \text { latij }
$$

where Yij denotes the response variable, $b 0, b 1$, and $b 2$ represent the fixed-effect parameters of the model, and $u i 0$ and $u i 1$ represent the ran- significantly different for the moving and static hands $\left(t_{(6)}=\right.$ $-4.177, p=0.006$; two-tailed paired-samples $t$ test) with a $13 \%$ of reduction in apparent time for the right hand $\left(t_{(6)}=-7.246\right.$, $p<0.0001$, two-tailed one-sample $t$ test, $\left.\mathrm{H}_{0}: \mu=150 \mathrm{~ms}\right)$ and virtually veridical time for the left hand $\left(t_{(6)}=0.320, p=0.759\right.$; two-tailed one-sample $t$ test, $\mathrm{H}_{0}: \mu=150 \mathrm{~ms}$; Fig. $3 C$, left). However, the precision thresholds do not significantly differ between 
A

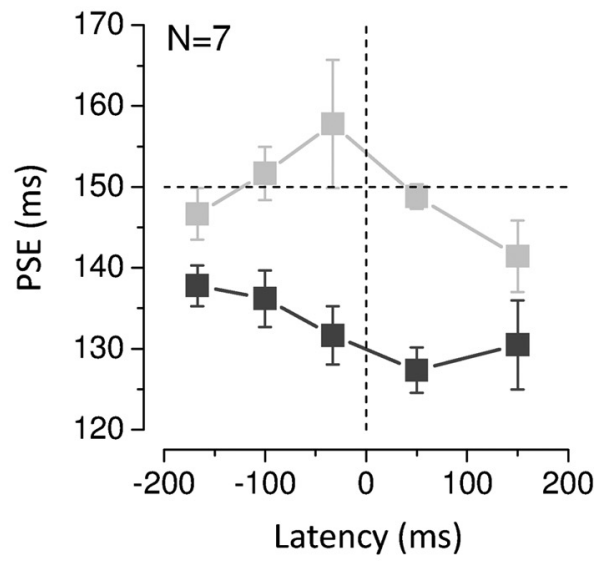

B

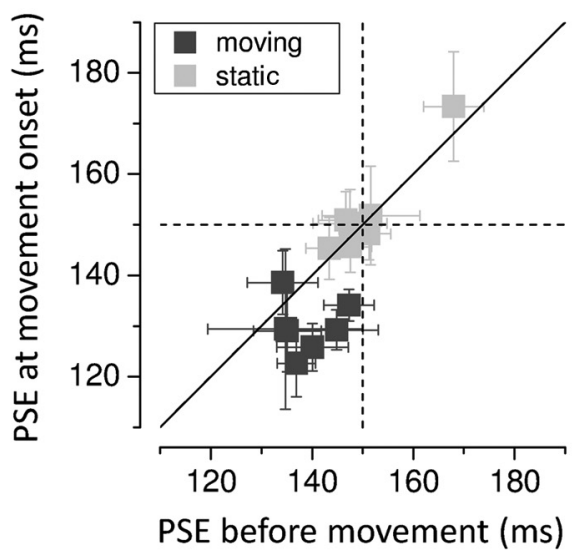

Figure 4. A, Average PSEs as a function of stimulus presentation time. The dashed horizontal line indicates physical duration; the dashed vertical line indicates movement onset time. Error bars represent SEM. $\boldsymbol{B}$, PSES around movement onset time (average \pm SD latency $=35 \pm 8$ $\mathrm{ms}$ ) plotted against those early before movement (average \pm SD latency $=-216 \pm 21 \mathrm{~ms}$ ) for the moving (black) and stationary hand (light gray; results for all subjects). The PSEs early before movement were calculated on the 60 trials with the earliest stimulus presentation times collected for each subject (starting from $-300 \mathrm{~ms}$ ), whereas the PSEs around movement onset included 60 trials up to stimulus latencies of $75 \mathrm{~ms}$. The vertical and horizontal dashed line represents physical duration. The diagonal shows equal perceived time early before and around movement onset. Error bars represent SEM.

the moving and stationary hand $\left(t_{(6)}=-0.609, p=0.565\right.$; twotailed paired-samples $t$ test), being $\sim 30 \mathrm{~ms}$ on average (Fig. $3 C$, right).

To investigate the temporal dynamics of the time compression effect, we took advantage of the existing variability in stimulus latency, defined as the time between the center of the test interval and movement onset (the average latencies \pm SD ranged from $-24 \pm 197 \mathrm{~ms}$ to $285 \pm 350 \mathrm{~ms}$ depending on the individual reaction times). First, we considered a latency window comprised between $-200 \mathrm{~ms}$ and $+200 \mathrm{~ms}$. We grouped data in five bins centered on $-167,-100,-33,50,150 \mathrm{~ms}$ so that each bin contained at least 30 trials for each subject and hand. Figure 4 shows the across-subjects average of the PSEs for each hand and bin. A two-way repeated-measures ANOVA on the PSEs with hand and latency as factors confirmed that time intervals appear shorter for the moving compared with the static hand $\left(F_{(1,6)}=16.666, p=\right.$ 0.006; main effect of factor hand). This analysis yielded also a marginally significant interaction between factor hand and latency $\left(F_{(4,24)}=2.624, p=0.06\right)$ reflecting the observation that
Moving hand Static hand

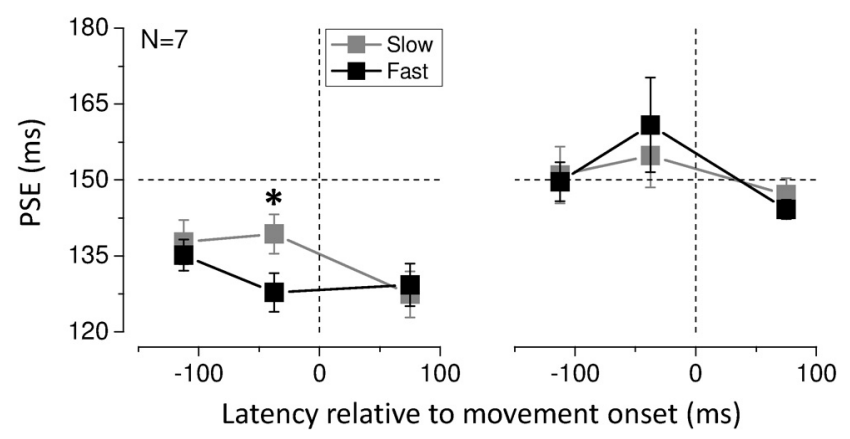

Figure 5. Average PSEs for stimuli presented to the moving and stationary hand as a function of stimulus presentation time, when fast and slow hand movements were executed. The dashed horizontal line indicates veridical duration; the dashed vertical line indicates movement onset time. Error bars represent SEM. The star represents statistical significance $(\alpha=0.05)$ of the difference between PSEs for fast and slow movements when the center of the test intervals fell within -75 and $0 \mathrm{~ms}$ (relative to movement onset).

perceived time for the right hand shows the tendency to shorten as the time of movement approaches (Fig. 4A). A seemingly opposite trend is observed for the left hand. However, the variation over time of the mean PSEs for the stationary hand is mainly due to the results of one subject (Fig. $4 B$ ), as indicated by the very large associated errors. The differential temporal modulation of the effect for the moving and stationary hand is confirmed by further analysis. Given the great variability in the individual reaction times, we could not average the results across subjects for stimulus latencies earlier than $\sim 170 \mathrm{~ms}$ relative to movement onset. To deal with the individual variation in dynamics, we compared the PSEs at the earliest stimulus latencies for each subject (that varied depending on the individual reaction times) with the PSEs around movement onset. Figure $4 B$ shows the change in perceived duration in trials corresponding to the earliest latencies observed for each subject (average \pm SD latencies varied from $-239 \pm 28 \mathrm{~ms}$ to $-142 \pm 29 \mathrm{~ms}$ depending on the subject; grand average $\pm \mathrm{SD}=-216 \pm 21 \mathrm{~ms}$ ) against the compression occurring around movement onset (grand average \pm SD latency $=$ $35 \pm 8 \mathrm{~ms}$ ) for the two hands. The pattern of results for the two hands is strikingly different. The data for the moving hand (black squares), with only one exception, are gathered below the equality line (diagonal) indicating a progressive reduction in perceived time during the motor preparatory period. On the contrary, the data for the stationary hand lie on the equality line indicating no change in perceived time as a function of stimulus latency. The two-way ANOVA for repeated measures with hand and latency (two latency bins; Fig. $4 B$ ) as within-subject factors confirmed the significant difference in perceived time between the two hands $\left(F_{(1,6)}=17.767, p=0.006\right)$ and most importantly yielded a significant interaction between hand and latency $\left(F_{(1,6)}=\right.$ 9.396, $p=0.022$ ). Moreover, to analyze the time course of the effect without binning the data we performed separate GLMM analyses for the moving and stationary hand including all trials with stimulus latencies up to $400 \mathrm{~ms}$ before movement onset (see Materials and Methods). For the right moving hand, we found a statistically significant effect for the factors probe $\left(b_{0}=0.0622\right.$, $p<0.0001)$ and latency $\left(b_{1}=0.0023, p=0.03\right)$. The positive sign of the coefficient for the latency factor indicates that apparent time progressively shortens as stimulus presentation time approaches the onset of the hand movement. No significant effect of latency was found for the left stationary hand $\left(b_{0}=0.0636, p<\right.$ 


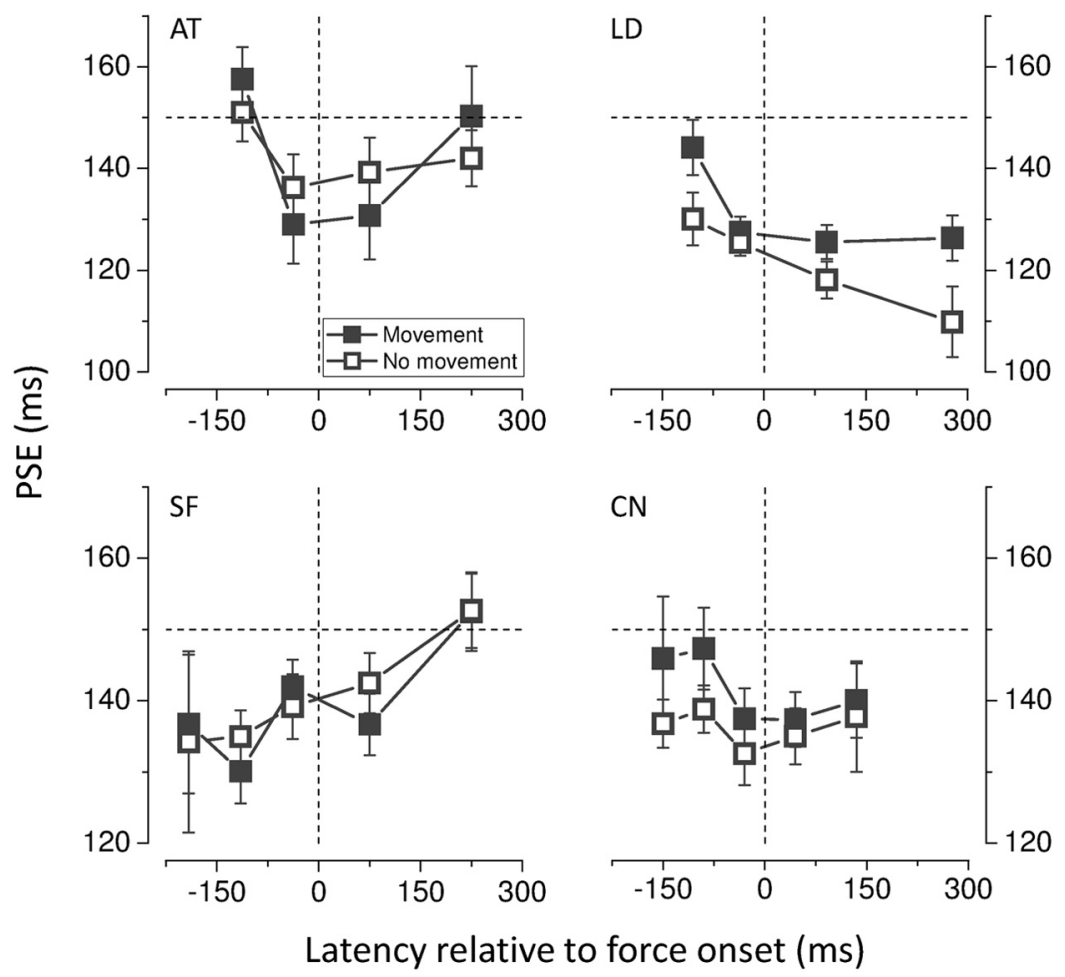

Figure 6. Individual PSEs for the movement and isometric contraction condition as a function of stimulus presentation time relative to force onset time. The dashed horizontal line indicates physical test duration; the dashed vertical line indicates force onset time. Error bars represent SEM.

0.0001 and $b_{1}=-0.0013, p=0.212$ for the probe and latency factors respectively), indicating that time compression is not due to motor preparation per se but it is specific for the motor effector.

The amplitude and duration of the arm movements performed by the participants during the experiment were $17 \pm 1 \mathrm{~cm}$ and $498 \pm 23 \mathrm{~ms}$ on average, which matched reasonably well the requirements. Arm velocity profiles were bell-shaped with peak velocity of $603 \pm 22 \mathrm{~mm} / \mathrm{s}$ reached $\sim 230 \pm 9 \mathrm{~ms}$ on average (mean $\pm \mathrm{SE}$ ). Although rather small, we exploited the across-trial variability in the movement kinematics to test whether the time compression effect is influenced by some characteristics of the movement. Individual data were analyzed separately depending on whether movement velocity was higher or lower than the median of the velocity distribution for each latency bin in the most critical time window (from -150 to $+150 \mathrm{~ms}$ ).

Average PSEs are reported in Figure 5 for the moving and stationary hands. Interestingly, faster movements caused a stronger compression of time for the stimuli delivered to the moving hand, only in the short moment just preceding movement onset $\left(\sim-40 \mathrm{~ms} ; t_{(6)}=3.005, p=0.024\right.$; two-tailed paired-samples $t$ test) when a difference in velocity between the two datasets was not reached yet $\left(t_{(6)}=-1.778, p=0.126\right.$; two-tailed pairedsamples $t$ test), pointing to an influence of the preparation for movement. No other significant difference was reported between fast and slow movements for either the moving or the static hand.

To understand the nature of the motor signals that may cause the observed temporal distortion we tested a further condition in which no movement was executed, but subjects were asked to produce a transient force against a block. The pattern of results obtained when subjects simply contracted hand muscles closely mirrors that obtained when they actually moved their hand. PSEs for the four tested subjects are shown in Figure 6 as a function of stimulus latency relative to force onset time. Regardless of the individual differences in the strength and time course of the effect, all subjects show a similar reduction in perceived time with similar temporal dynamics in the condition of real movement compared with the condition of isometric contraction. A $2 \times 4$ ANOVA for repeated measures was conducted on the PSE data with movement (movement vs isometric contraction) and latency (two latency bins before and after force onset) as within-subjects factors yielding no significant main effect of factor movement $\left(F_{(1,3)}=1.28, p=0.34\right)$ as well as no significant interaction effect $\left(F_{(3,9)}=2.14, p=0.165\right)$.

\section{Experiment 2: effect of tactile suppression on perceived time}

Body movement as well as isometric contraction is known to be preceded and accompanied by a transient damping in tactile sensitivity which is generally referred to as tactile suppression (Williams et al., 1998). There is also evidence that temporal judgments are affected by the strength of sensory stimulation (for example, by visual contrast and luminance), with weaker and masked stimuli appearing shorter (Terao et al., 2008). In Experiment 1 to control the detectability of both taps in the pair, we asked subjects to indicate whether they perceived one or two stimuli in each trial. The overall number of trials where unclear percepts were reported was very low $(<1 \%)$ and they were discarded from analysis. In addition, to be sure that any effect on perceived time observed during movement was not a mere consequence of tactile suppression, we performed a control experiment on five of the seven naive subjects who had taken part in the main experiment. First, we measured the amount of reduction in the perceived intensity of tactile stimuli delivered to the motor effector at different times with respect to the movement (from $\sim 200$ ms before to $\sim 100 \mathrm{~ms}$ after force onset time). The experimental procedure was identical to the Experiment 1 (movement condition), except that subjects had to compare the perceived intensity of the tactile stimulations. The intensity-matching experiment yields a similar pattern of results for all the five tested subjects. In agreement with previous reports (Williams and Chapman, 2000), perceived stimulus intensity on the hand effector is attenuated both before and during movement. The attenuation effect gradually increases within the temporal window investigated, reaching its maximal value for stimulus latencies of $\sim 0-100$ ms after force onset time (PSE $=1.8 \pm 0.1 \mathrm{~V}$ on average; Fig. $7 A$ ). Test stimuli of intensity values corresponding to the individual PSEs obtained in the matching experiment for the 0-100 ms stimulus latencies (matched condition; see Materials and Methods), intermingled with stimuli of standard intensity (baseline condition), were used in the temporal control experiment and delivered to the right stationary hand. Data for two representative subjects (same subjects reported in Fig. 2) are plotted in Figure $7 C$, showing no difference in apparent time between the matched and the baseline condition, as indicated by the nearly overlapping psychometric functions, and strong compression of 
time for the movement condition. Temporal judgments between the matched and baseline condition were not significantly different $\left(t_{(4)}=-1.038, p=0.358\right.$; twotailed paired-samples $t$ test), whereas perceived time during movement (results from Experiment 1) is significantly reduced with respect to the matched $\left(t_{(4)}=\right.$ 3.056, $p=0.038$; two-tailed pairedsamples $t$ test; Fig. $7 B$ ), as well as to the baseline condition $\left(t_{(4)}=-7.776, p=\right.$ 0.001 ; two-tailed paired-samples $t$ test). We conclude that the reduction in perceived tactile intensity during movement cannot account for the reduction in perceived duration. The lack of difference between the baseline and the matched condition further supports the conclusion that the amount of tactile attenuation during movement (at least for the stimulus intensities used in our study) is not sufficient to induce any significant bias in apparent time.

\section{Discussion}

We show that time intervals marked by tactile stimuli appear shorter when hand movements are prepared and executed. Our result corroborates recent evidence of a tight link between time perception and action (Yarrow et al., 2001; Haggard et al., 2002; Morrone et al., 2005; Hagura et al., 2012; Tomassini et al., 2012) and shows a selective and anticipatory movementrelated distortion of time. Critically, at the moments around action initiation, time compression is restricted to the motor effector. Duration is misjudged only when tactile stimuli are delivered to the hand that is about to move and not when delivered to the other, stationary hand, indicating that movement preparation selectively disrupts temporal processing on the motor effector.

Attention is known to exert powerful effects on time perception (Tse et al., 2004), typically causing temporal expansion when focused on the timing task and contraction when directed elsewhere. There are many ways by which attentional factors might influence duration judgments in our task but some of them can be dismissed very easily. The compression of time cannot be caused by a general decrease of attention due to the dual-task performance (Fraisse, 1984; Brown, 1985). In fact, that would produce comparable effects for the moving and stationary hand (as participants were equally engaged in the same attentionaldemanding task) rather than selective effects, as we found. Time compression cannot be due to a stronger attentional allocation to the motor effector (Forster and Eimer, 2007), given that salient and attended stimuli generate expansion not compression of time (Block and Zakay, 1997; Tse et al., 2004). Eye and hand movements have been shown to be coupled with predictive shifts of attention to the movement target and to the motor effector (Eimer et al., 2006; Juravle and Deubel, 2009). However, our effect is in the opposite direction from what is expected on the basis of selective attention. Expansion, rather than compression, would be predicted for the stimuli delivered to the moving hand subjects of Fig. 2) are shown.
B
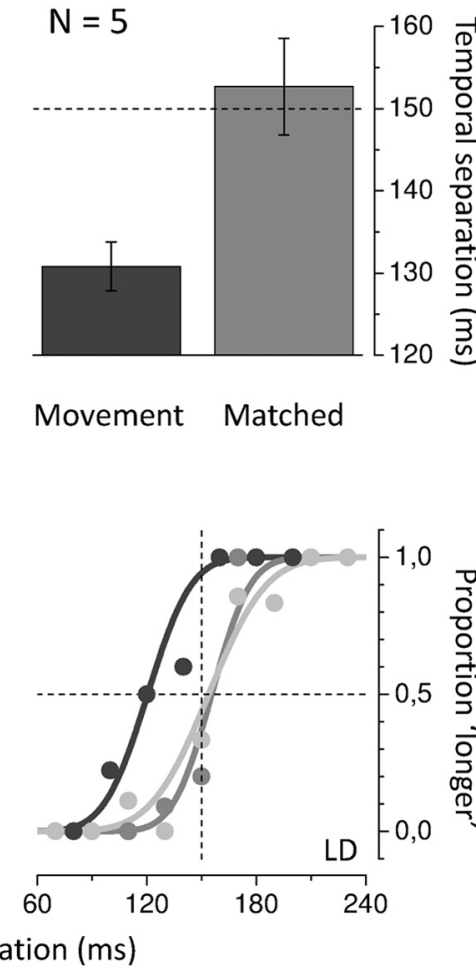

Figure 7. Control experiment $\boldsymbol{A}$, Average PSEs for the intensity-matching experiment plotted as a function of stimulus presenafter force onset) that was used to determine the matching-intensity and then averaged across the same five subjects who took part in the control experiment. The dashed horizontal line indicates physical duration (150 ms). Error bars represent SEM. $\boldsymbol{C}$ Psychometric functions showing the proportion of trials where the probe was judged to be longer than the test in the movement (black), intensity-matched (gray), and baseline (light gray) condition. Data for two representative subjects (SC and LD; same

if the change in apparent time was driven by a motor-related shift of attention toward the effector. Moreover, tactile stimulations occurred with equal probability on either hand, ruling out any systematic bias in somatosensory attention or prior-entry like effects. Therefore, we could exclude that the time compression merely reflects dynamic allocation of motor or spatial attention. Finally, time compression is unlikely to be ascribed to an unspecific increase in noise, owing for example to movement-induced masking effects, given that we observed the same precision in temporal judgments for the various conditions.

A general decrease in tactile sensitivity is known to occur early before and during voluntary executed movements (Angel and Malenka, 1982; Milne et al., 1988; Williams et al., 1998; Shergill et al., 2003). This phenomenon, known as tactile suppression, has probably a central component, as it precedes movement onset and even electromyographic activity (Williams et al., 1998). Indeed, it has been recently argued that time underestimation might result from weak responses to transient signals, at least in the visual system (Terao et al., 2008). We quantified the amount of tactile attenuation during movement and then measured perceived time in static conditions for stimuli whose physical intensity matched the perceived intensity during movement. The results indicate that apparent time for these low-intensity matched stimuli is virtually veridical. Thus, the compression of time observed is most likely a genuine movement-related effect and not simply a byproduct of suppression. However, in the pres- 
ent study we did not measure time compression during movement for stimuli of different intensities and uncontrolled order effects or learning may have influenced our results. Thus, because not all of the methodological decisions underlying this work were finalized before the processes of data collection and analysis, our findings will benefit from confirmatory replication.

A growing number of evidence shows a close relation between motor and temporal processing. Within the large and complex network of areas that has been related to timing processes, "purely" motor circuits are indeed primarily represented (Lewis and Miall, 2003; Coull et al., 2004; Macar et al., 2006; Teki et al., 2011; Bueti et al., 2012; Coull et al., 2012). We find that the amount of time compression during motor preparation is modulated by the velocity of the forthcoming movement, with greater compression associated with preparation for faster movements. This suggests that high speed does not act by simply increasing the noise level in reafferent signals transmission possibly enhancing masking effects, as this would affect temporal judgments during motor execution rather than during preparation when the effector is still stationary. Similarly, the modulation by movement speed is also unlikely to be ascribed to suppression, given that tactile suppression is not sufficient to induce the perceived time compression. Rather, this result is a strong indication that the motor preparatory activity may play a direct role in the temporal effects reported here. Indeed, it has been shown that the velocity of upcoming movements is codified by the preparatory motor activity of neurons in both premotor (PMd) and primary motor areas (M1; Churchland et al., 2006). Not surprisingly, also the saccade-related effects on visual time are similarly modulated by eye movement velocity (Ostendorf et al., 2007), outlining further connections between the visual and tactile phenomena.

To understand better the nature and origin of the temporal distortion accompanying hand movements we tested also an isometric condition where motor processing should be free of any spatial transformation that serves the construction of a motor plan. Our results show that motor efference, with no associated spatial displacement of any body part, induces comparable compression of perceived time as actual movement. This suggests that the perceptual effects on time might be mediated by descending motor signals and not by ascending reafferent signals, which are largely minimized in the isometric contraction condition. It also indicates that the motor signal responsible for these effects is most probably a high-level signal in the motor hierarchy, generated at an initial stage of motor processing when the intentionto-move is formulated. The haptic system provides an exclusive opportunity to test whether the displacement of the body is a necessary condition to induce the perceptual distortions. Previous studies have exploited this possibility investigating the tactile suppression and obtaining similar results during contraction and movement execution (Post et al., 1994; Williams and Chapman, 2002; Wasaka et al., 2005). Thus, both compression and suppression can take place without actual displacement of the body and both precede movement onset. The two phenomena show evident commonalities suggesting they might distinctively contribute to a similar brain function.

The changes in apparent time that we observe for tactile stimuli presented at the time of hand movements closely resemble the perisaccadic distortions of visual time. Several similarities can be traced between the two phenomena: for both modalities, the distortion has an anticipatory nature, preceding movement onset, and it is modality- or effector-specific. The compression of tactile time that we report differs however in some respects from its saccadic counterpart. It is in fact smaller and has a slower tempo- ral dynamics. Apparent visual time is reduced up to half of its physical length starting from $\sim 150 \mathrm{~ms}$ before saccadic onset, whereas the decrease in tactile time begins slightly earlier $(\sim 200$ $\mathrm{ms}$, although with some variation across subjects) and reaches a maximum of $15 \%$. The faster dynamics of the visual effect might reflect the faster time course of the motor preparatory activity within the oculomotor system. Another phenomenon common to the visual and tactile domain is the apparent temporal inversion of two separate stimuli that take place during the planning of hand (Hermosillo et al., 2011) and eye movements (Morrone et al., 2005; Kitazawa et al., 2008). Time order judgments are also susceptible to hand posture and body schema manipulations, implying that time alterations are dependent on spatial representations (Morrone et al., 2010) built up through multimodal processes that order the flow of sensory events in time (Kitazawa et al., 2008).

The perisaccadic changes in visual time have been linked to the mechanisms that achieve visual stability against the abrupt shift of the retinal image consequent to eye movement (Binda et al., 2009; Burr and Morrone, 2011; Cicchini et al., 2013), probably mediated by the predictive remapping of visual receptive fields (Duhamel et al., 1992; Sommer and Wurtz, 2002). Tactile information must be also remapped across hand movements (or skin somatotopy) to determine the position and shape of the touched objects. As we move our hands, the relevant tactile features of the explored surfaces (e.g., edges, bumps, and dips) will successively stimulate different portions of the skin (for example different fingertips). To correctly interpret this time-varying signal and accurately reconstruct the object properties, information about the upcoming movements must be available. This knowledge might be used in advance both to filter out irrelevant signals from the incoming sensory flow, as well as to appropriately remap tactile signals across movements.

The compression of apparent time and the suppression might thus reflect a complex and multifaceted set of mechanisms that optimize sensory-motor functions.

Whether the distortions of time and space for tactile stimuli at the moment of action (Dassonville, 1995; Watanabe et al., 2009) share a common origin, as it has been suggested for vision, is an intriguing still open question. The present results might suggest a common strategy within different sensory-motor domains that may serve the maintenance of perceptual stability during movement.

\section{References}

Angel RW, Malenka RC (1982) Velocity-dependent suppression of cutaneous sensitivity during movement. Exp Neurol 77:266-274. CrossRef Medline

Binda P, Cicchini GM, Burr DC, Morrone MC (2009) Spatiotemporal distortions of visual perception at the time of saccades. J Neurosci 29:1314713157. CrossRef Medline

Block RA, Zakay D (1997) Prospective and retrospective duration judgments: a meta-analytic review. Psychon Bull Rev 4:184-197. CrossRef Medline

Brown SW (1985) Time perception and attention: the effects of prospective versus retrospective paradigms and task demands on perceived duration. Perception and psychophysics 38:115-124. CrossRef Medline

Bueti D, Lasaponara S, Cercignani M, Macaluso E (2012) Learning about time: plastic changes and interindividual brain differences. Neuron 75: 725-737. CrossRef Medline

Burr DC, Morrone MC (2011) Spatiotopic coding and remapping in humans. Philos Trans R Soc Lond B Biol Sci 366:504-515. CrossRef Medline

Churchland MM, Santhanam G, Shenoy KV (2006) Preparatory activity in premotor and motor cortex reflects the speed of the upcoming reach. J Neurophysiol 96:3130-3146. CrossRef Medline

Cicchini GM, Binda P, Burr DC, Morrone MC (2013) Transient spatiotopic 
integration across saccadic eye movements mediates visual stability. J Neurophysiol 109:1117-1125. CrossRef Medline

Coull JT, Vidal F, Nazarian B, Macar F (2004) Functional anatomy of the attentional modulation of time estimation. Science 303:1506-1508. CrossRef Medline

Coull JT, Hwang HJ, Leyton M, Dagher A (2012) Dopamine precursor depletion impairs timing in healthy volunteers by attenuating activity in putamen and supplementary motor area. J Neurosci 32:16704-16715. CrossRef Medline

Dassonville P (1995) Haptic localization and the internal representation of the hand in space. Exp Brain Res 106:434-448. Medline

Duhamel JR, Colby CL, Goldberg ME (1992) The updating of the representation of visual space in parietal cortex by intended eye movements. Science 255:90-92. CrossRef Medline

Eimer M, Van Velzen J, Gherri E, Press C (2006) Manual response preparation and saccade programming are linked to attention shifts: ERP evidence for covert attentional orienting and spatially specific modulations of visual processing. Brain Res 1105:7-19. CrossRef Medline

Forster B, Eimer M (2007) Covert unimanual response preparation triggers attention shifts to effectors rather than goal locations. Neurosci Lett 419: 142-146. CrossRef Medline

Fraisse P (1984) Perception and estimation of time. Annu Rev Psychol 35: 1-36. CrossRef Medline

Gibson JJ (1962) Observations on active touch. Psychol Rev 69:477-491. CrossRef Medline

Haggard P, Clark S, Kalogeras J (2002) Voluntary action and conscious awareness. Nat Neurosci 5:382-385. CrossRef Medline

Hagura N, Kanai R, Orgs G, Haggard P (2012) Ready steady slow: action preparation slows the subjective passage of time. Proc Biol Sci 279:43994406. CrossRef Medline

Helmholtz (1866) Concerning the perceptions in general. Treatise on physiological optics, Vol 3, Ed 3. J.P.C. Southall 1925 Opt Soc Am, Section 26.

Hermosillo R, Ritterband-Rosenbaum A, van Donkelaar P (2011) Predicting future sensorimotor states influences current temporal decision making. J Neurosci 31:10019-10022. CrossRef Medline

Jackson SR, Parkinson A, Pears SL, Nam SH (2011) Effects of motor intention on the perception of somatosensory events: a behavioural and functional magnetic resonance imaging study. Q J Exp Psychol (Hove) 64: 839-854. CrossRef Medline

Juravle G, Deubel H (2009) Action preparation enhances the processing of tactile targets. Exp Brain Res 198:301-311. CrossRef Medline

Kitazawa S, Moizumi S, Okuzumi A, Saito F, Shibuya S, Takahashi T, Wada M, Yamamoto S (2008) Reversal of subjective temporal order due to sensory and motor integrations. In: Sensorimotor foundations of higher cognition attention and performance XXII (Haggard P, et al., eds), pp 73-97. Oxford: Oxford UP.

Lappe M, Awater H, Krekelberg B (2000) Postsaccadic visual references generate presaccadic compression of space. Nature 403:892-895. CrossRef Medline

Lewis PA, Miall RC (2003) Brain activation patterns during measurement of sub- and supra-second intervals. Neuropsychologia 41:1583-1592. CrossRef Medline

Macar F, Coull J, Vidal F (2006) The supplementary motor area in motor and perceptual time processing: fMRI studies. Cogn Process 7:89-94. CrossRef Medline

Maij F, de Grave DD, Brenner E, Smeets JB (2011) Misjudging where you felt a light switch in a dark room. Exp Brain Res 213:223-227. CrossRef Medline

Medendorp WP (2011) Spatial constancy mechanisms in motor control. Philos Trans R Soc Lond B Biol Sci 366:476-491. CrossRef Medline

Milne RJ, Aniss AM, Kay NE, Gandevia SC (1988) Reduction in perceived intensity of cutaneous stimuli during movement: a quantitative study. Exp Brain Res 70:569-576. Medline

Morrone MC, Ross J, Burr DC (1997) Apparent position of visual targets during real and simulated saccadic eye movements. J Neurosci 17:79417953. Medline
Morrone MC, Ross J, Burr D (2005) Saccadic eye movements cause compression of time as well as space. Nat Neurosci 8:950-954. CrossRef Medline

Morrone MC, Cicchini M, Burr DC (2010) Spatial maps for time and motion. Exp Brain Res 206:121-128. CrossRef Medline

Moscatelli A, Mezzetti M, Lacquaniti F (2012) Modeling psychophysical data at the population-level: the generalized linear mixed model. J Vis 12(11):26 1-17. CrossRef Medline

Ostendorf F, Fischer C, Finke C, Ploner CJ (2007) Perisaccadic compression correlates with saccadic peak velocity: differential association of eye movement dynamics with perceptual mislocalization patterns. J Neurosci 27:7559-7563. CrossRef Medline

Post LJ, Zompa IC, Chapman CE (1994) Perception of vibrotactile stimuli during motor activity in human subjects. Exp Brain Res 100:107-120. Medline

Ross J, Morrone MC, Burr DC (1997) Compression of visual space before saccades. Nature 386:598-601. CrossRef Medline

Rucci M, Iovin R, Poletti M, Santini F (2007) Miniature eye movements enhance fine spatial detail. Nature 447:851-854. CrossRef Medline

Shergill SS, Bays PM, Frith CD, Wolpert DM (2003) Two eyes for an eye: the neuroscience of force escalation. Science 301:187. CrossRef Medline

Sommer MA, Wurtz RH (2002) A pathway in primate brain for internal monitoring of movements. Science 296:1480-1482. CrossRef Medline

Teki S, Grube M, Kumar S, Griffiths TD (2011) Distinct neural substrates of duration-based and beat-based auditory timing. J Neurosci 31:38053812. CrossRef Medline

Terao M, Watanabe J, Yagi A, Nishida S (2008) Reduction of stimulus visibility compresses apparent time intervals. Nat Neurosci 11:541-542. CrossRef Medline

Tomassini A, Gori M, Burr D, Sandini G, Morrone MC (2012) Active movement restores veridical event-timing after tactile adaptation. J Neurophysiol 108:2092-2100. CrossRef Medline

Tse PU, Intriligator J, Rivest J, Cavanagh P (2004) Attention and the subjective expansion of time. Percept Psychophys 66:1171-1189. CrossRef Medline

Vaziri S, Diedrichsen J, Shadmehr R (2006) Why does the brain predict sensory consequences of oculomotor commands? Optimal integration of the predicted and the actual sensory feedback. J Neurosci 26:4188-4197. CrossRef Medline

Wasaka T, Nakata H, Kida T, Kakigi R (2005) Changes in the centrifugal gating effect on somatosensory evoked potentials depending on the level of contractile force. Exp Brain Res 166:118-125. CrossRef Medline

Watanabe J, Nakatani M, Ando H, Tachi S (2009) Haptic localizations for onset and offset of vibro-tactile stimuli are dissociated. Exp Brain Res 193:483-489. CrossRef Medline

Williams SR, Chapman CE (2000) Time course and magnitude of movement-related gating of tactile detection in humans: II. Effects of stimulus intensity. J Neurophysiol 84:863-875. Medline

Williams SR, Chapman CE (2002) Time course and magnitude of movement-related gating of tactile detection in humans: III. Effect of motor tasks. J Neurophysiol 88:1968-1979. Medline

Williams SR, Shenasa J, Chapman CE (1998) Time course and magnitude of movement-related gating of tactile detection in humans: I. Importance of stimulus location. J Neurophysiol 79:947-963. Medline

Yamamoto S, Kitazawa S (2001a) Reversal of subjective temporal order due to arm crossing. Nat Neurosci 4:759-765. CrossRef Medline

Yamamoto S, Kitazawa S (2001b) Sensation at the tips of invisible tools. Nat Neurosci 4:979-980. CrossRef Medline

Yarrow K, Haggard P, Heal R, Brown P, Rothwell JC (2001) Illusory perceptions of space and time preserve cross-saccadic perceptual continuity. Nature 414:302-305. CrossRef Medline

Yoshioka T, Craig JC, Beck GC, Hsiao SS (2011) Perceptual constancy of texture roughness in the tactile system. J Neurosci 31:17603-17611. CrossRef Medline 